\title{
The effects of multiple layers feed-forward neural network transfer function in digital based ethiopian soil classification and moisture prediction
}

\author{
Belete Biazen Bezabeh, Abrham Debasu Mengistu \\ Faculty of Computing, Bahir Dar Institute of Technology, Bahir Dar University, Ethiopia
}

\begin{abstract}
Article Info
Article history:

Received Apr 5, 2019

Revised Feb 22, 2020

Accepted Mar 1, 2020

Keywords:

FFNN

Gaussian transfer function

ReLU transfer function

Sigmoid transfer function

Tanh transfer function

ABSTRACT

In the area of machine learning performance analysis is the major task in order to get a better performance both in training and testing model. In addition, performance analysis of machine learning techniques helps to identify how the machine is performing on the given input and also to find any improvements needed to make on the learning model. Feed-forward neural network (FFNN) has different area of applications, but the epoch convergences of the network differs from the usage of transfer function. In this study, to build the model for classification and moisture prediction of soil, rectified linear units (ReLU), Sigmoid, hyperbolic tangent (Tanh) and Gaussian transfer function of feed-forward neural network had been analyzed to identify an appropriate transfer function. Color, texture, shape and brisk local feature descriptor are used as a feature vector of FFNN in the input layer and 4 hidden layers were considered in this study. In each hidden layer 26 neurons are used. From the experiment, Gaussian transfer function outperforms than ReLU, sigmoid and tanh transfer function. But the convergence rate of Gaussian transfer function took more epoch than ReLU, Sigmoid and tanh.
\end{abstract}

Copyright () 2020 Institute of Advanced Engineering and Science. All rights reserved.

\author{
Corresponding Author: \\ Belete Biazen Bezabeh, \\ Faculty of Computing, \\ Bahir Dar Institute of Technology, Bahir Dar University, \\ P.O.BOX 26, Bahir Dar Ethiopia. \\ Email: Belete.Biazen@bdu.edu.et
}

\section{INTRODUCTION}

Agricultural production has been highly dependent on natural resources for centuries. According to FAO, soil quality maintenance is important for agricultural annual cropping, environmental and economic sustainability. In related, a gradual turn down in soil quality has a negetive impact on plant growth and annual or seasonal, grain quality, production costs and finally it leads to risk of soil erosion [1]. The field of Computer vision and digital image processing (DIP) is continuously evolving and is finding many applications in several fields. Soil classification and moisture level prediction is an important aspect of geotechnical engineering which has been given a great amount of attention since the past few years. Vision data in geosciences particularly soil classification and moisture level prediction are commonly used. In order to use, it require processing and measurement schemes that range from small microscopic scales to large remote sensing scales. They focused mainly to the first category and specifically in images of thin soil sections. In [2], the authors stated that soil micro morphology in related to soil science, is the description, interpretation, and measurement of components, features, and fabrics in soils at a microscopic level. Besides, in the paper the author has focused on texture analysis and segmentation techniques of the given soil type.

The technology of image processing advancement is gradually finding applications in different problem domains like in the areas of agriculture, health and others which needs to be supported by computer 
vision. In the areas of machine learning, human operator efforts are being replaced with automated systems, this is due to, as human operations are usually inconsistent and non efficient. Automated systems in most cases are faster and more precise than the traditional human efforts. However, there are some basic infrastructures that must necessarily be in place in automation [3].

In [4], due to sensor nodes have decreased in size and are much cheaper, it can be appliecd in the emergence of many new civilian applications from environment monitoring to vehicular and body sensor networks. Inspites the authors have used sensor for monitoring environments and achieved promising result. They didn't shown the effects on transfer function in related with soil classiofication and and moisture prediction. In addition, the authors stated in [5], sensor-based technologies can be applied in construction safty management. But there is still a gap of model to identify a better transfer function for classification of soil and moisture predeiction.

A study conducted by [6] to estimate soil crack for moisture analysis, from the experiment $72.7 \%$ is archived. In the study, they have used texture and color as a feature vector and support vector machine to estimate crack. Even though, they achieved $72.7 \%$, kerenel function of SVM had not been experimented to increase the performance. According to [7], they stated that to avoid agricultural product both quality and quantity loss, soil characteristics identification and classification is crutial task.

Soil characterization and classification using computer vision \& sensor network approach has been studied. In their study the authors have used Gravity Analog Soil Moisture Sensor with arduino-uno and image processing as techniques to achieve the objective [8]. But, there is a gap to identify suitable learning function of neural network for better convergence and accuracy.

In [9], the authors have used computer vision for limestone rock-type classification using probabilistic neural network. In this paper, a computer vision based rock type classification system is proposed without human intervention using the probabilistic neural network (PNN). In this research paper the authors are used the color histogram features as an input. In the paper the color image histogram based features includes weighted mean, skewness and kurtosis features are extracted for all three color space red, green, and blue. In this paper, a total nine features are used as input for the PNN classification model. Then they found out the error rate for identification is below 6\%. In [10], the authors have presented soil image segmentation and texture analysis using computer vision approach. The author proposed joint image segmentation methods for soil images and feature measurements.

Soil texture classification algorithm using RGB characteristics of soil images has been done. The authors found that soil texture has traditionally been determined in the laboratory using pipette and hydrometer methods that require a considerable amount of time, labor, and expense. In the paper, soil texture classification using RGB histograms was investigated to achieve the goal of the study. In this paper, when soils were classified using USDA soil texture classification, the laboratory method and image processing method produced the same results for $48 \%$ of the samples [11]. In [12], the authors have shown that detection of soil pore structure using an image segmentation approach. In this study, a density based clustering method on tomography sections of soil is considered.

In [13], the authors have studied determination of Soil $\mathrm{pH}$ by using Digital Image Processing Technique. In Agriculture sector the parameters like quantity and quality of product are the important measures from the farmers' point of view. The soil is recognized as one of the most valuable natural resource whose soil $\mathrm{pH}$ property used to describe the degree of acidity or basicity which affects nutrient availability and ultimately plant growth.

Image texture analysis and neural networks for characterization of uniform soils are studied. Supervised back-propagation neural network is used for this study. The authors have tested neural network with considerable accuracy [14]. Here, there is a gap to tune the learning function of neural network for analyzing the performance of the model.

In [15], the authors presented "Testing of Agriculture Soil by Digital Image Processing". This paper helps to determine the amount of fertilizer and $\mathrm{pH}$ of soil that must be applied. 80 soil samples their $\mathrm{pH}$ value tested in government soil testing Lab are considered in this study. In their work, when the software is tested the software gives $60-70 \%$ accuracy.

Wavelet analysis of soil reflectance for the characterization of soil properties has been studied. The authors have used Wavelet analysis, hyper spectral near-infrared (NIR) and mid-infrared (MIR) reflectance spectra of soil material to characterize the given soil [16]. In [17], the authors have used a novel method for 2-D Agricultural soil roughness characterization based on a laser scanning technique" presented laser profiler the determination of agricultural soil roughness. When tested with the RMS height $\mathrm{S}$ and correlation length $\mathrm{L}$ in $1 \mathrm{~m}$ x 0,3 m parcels with a 20-30\% error in heights and 1- 10\% error in horizontal lengths.

The application of image processing and analysis in plant root systems in soil has been done using imageJ plat form. ImageJ is a java version that is used to perform image analysis. The authors have used 
X-ray tomography 3D images [18]. In [19], they studied detection of soil liquefaction areas in case of Kantou region of Japan. In this paper, multi-temporal PALSAR coherence data is considered.

According to [20], moisture content in soil is one of the main component which plays important role in yield of crops. In this paper the authors focused on software development for soil moisture assessment. The main objective of the authors was to turn the manual process to a software application using image processing technique. Image of the soil with different moisture content are collected and preprocessed to remove the noise of source image. The authors have used color and texture feature vector as an input in soil moisture assessment software.

In [21], the authors stated that the farmers are suffering from the lack of rains and scarcity of water. In this paper, the main objective was to provide an automatic irrigation system thereby saving time, money and power of the farmer. In this work moisture sensors are considered anad installed on the field. Whenever there is a change in water content of soil these sensors sense the change gives an interrupt signal to the micro-controller For capturing the images, the phone camera is used and after processing the captured image the $\mathrm{PH}$ value of the soil is determined and accordingly crops or plants are suggested that can be grown in that field.

In [22], the authors focused on an urban road materials vision system using narrow band near infrared imaging. This paper proposed imaging indexes evaluation from experiment results to identify those urban road materials. The proposed multi-spectral imaging indexes were able to show the potential to classify the selected urban road materials, another approach may need to clearly distinguish between concrete and aggregates.

Data mining approach for soil moisture prediction has been studied. In this paper, the authors have used five different algorithms i.e. KNN, SVM, Logistic regression neural network and rule induction. Form the experiment the authors have found that KNN has a better performance than SVM and neural network [22].

In [23], they present an artificial neural network framework for predicting earth block performance prediction. In their study, they stated that earth block performance depends heavily on the qualities of soil used and it is important to identify the qualities of soil. But in this paper they have not seen soil classification and moisture level prediction.

In [24], the authors have pointed that the application of data mining towards to learning and classifying agricultural soil types. In their study, they have used naïve bayes, J48 and JRip techniques for classification of soil and predicting of PH values. Finally, form the experiment they have found that JRip has better performance than the other data mining techniques.

The combined approach of SOM and KNN for classifying agricultural soil has been studied. In the study, the authors have used color and texture feature vectors. From the experiment they achieved $79.8 \%$ result when SOM and KNN are used [25].

In [26], in this study, they have used Artificial neural network for predicting soil types. During their work a database consists of 120 soil samples which were collected from Shahrekord, Chahar Mahal and Bakhtiari province. Finally they have tested their model using neural network. The neural network used in this study has an input layer, an output layer and a hidden layer. The input layer consists of 7 variables and the output layer has only one parameter for predicting soil type and the hidden layer has 15 neurons. The number of nodes in the hidden layer was determined by trial and error.

In [27], they conducted a study related with moisture and organic matter prediction based on NIR reflectance sensor. In the study, the authors used SOM (self organizing map) for learning the pattern and prediction. From the experiment they have found that Standard errors of prediction for organic matter and soil moisture were 0.62 and $5.31 \%$, respectively.

In [28], they studied the application of machine learning techniques in soil classification. They consider only three type of soil (sand, clay, peat) and for machine learning side the authors have considered Artificial neural network (ANN), Decision tree (DT) and support vector machine (SVM) and WEKA software is used for classifying to their Corresponding class. From the experiment the authors have shown that ANN is better than DT and SVM. This study is limited in three classes and still there is a gap to show the moisture level.

Prediction of rainfall using image processing" the authors have used digital cloud images to predict rainfall. From this paper they stated that it is better to predict rainfall from digital cloud images rather than satellite images based on the cost factors and security issues. They have used wavelet and Cloud Mask Algorithm to capture the image for clustering K- means is used [29].

In [30], they studied about soil classification and quantitatively predict the temperature and moisture of the soil based on satellite image. In this research paper the authors have used 3 types of soils from the satellite. For classification and moisture prediction BPNN and LM were used. From the experiment they concluded that neural networks can be used as a paradigm in soil classification as well as in predicting

The effects of multiple layers feed-forward neural network transfer function in... (Belete Biazen Bezabeh) 
the quantity of soil moisture and temperature accurately, using remotely sensed microwave data, and thus helps achieve a proper crop management.

Detection of nutritional deficiencies in plants has been researched in [31]. In the paper they have used color and shape as feature vector and as a classifier KNN, SVM and deep learning has been considered. In [32], the authors have proposed soil characterization and classification using computer vision \& sensor network approach. BPNN has been used as a classifier and from the experiment they have got $89.7 \%$. The application of Back Propagation Neural Network to wards to breast cancer and lung cancer has been studied in [33]. In their work to enhance the accuracy of the classifer, genetic algorithms was used and a better result has been achieved.

In the area of machine learning performance analysis is the major task in order to get a better performance both in training and testing model. In addition, performance analysis of machine learning techniques helps to determine how the machine is performing and whether any improvments needed to made. Artificial neural network can be applied in different areas of sector but, their speed of learning and network complexity may differ from transfer function. The neural networks may learn patterns, but the inner ability to learn quickly the given pattern requires flexible transfer functions that are suitable for the problem to be solved. Different techniques have been proposed related with soil classification and moisture characterization. However, according to the litratures and to the best of our knowledge, no research has been conducted related to the performance analysis of transfer function in Ethiopian soil classification and moisture level prediction. Therefore, the main goal of this study is to find the suitable transfer function for Ethiopian Soil classification and moisture level prediction. In this study, rectified linear units (ReLU), sigmoid, Gaussian and hyperbolic tangent (tanh) transfer functions are tested to achieve the objective of this research.

\section{MODEL DESIGN}

As shown in Figure 1 soil classification and moisture level prediction model consists of three basic parts: image aquizition, Feature extraction and performance evaluation.

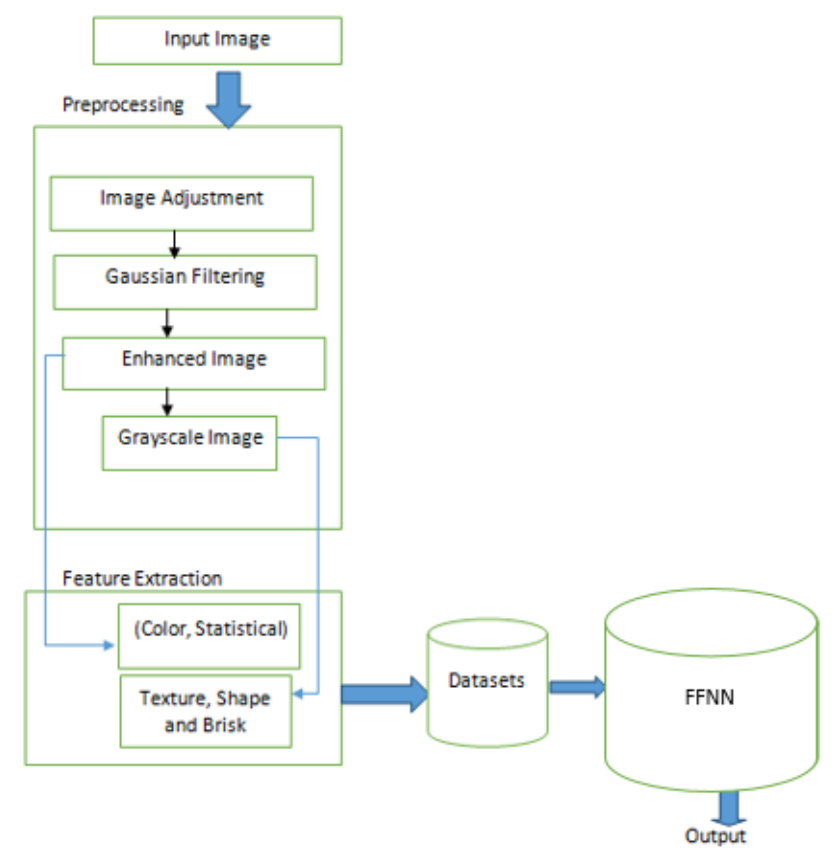

Figure 1. Model design (adopted from [8])

\subsection{Image acquisition}

In computer vision, image acquisition is the first part. In this study we used smart phone camera to the images in the form of JPEG (Joint Photographers Expert Groups) file format. In addition, 256 by 256 images size is considered for this study. The total of 6 group of soil and each having 90 images are considered for this study. That is, form these 540 images were captured at different places of Amhara regions of Ethiopia. 


\subsection{Image preprocessing}

Image processing is a technique that manipulates images in various ways to enhance image quality. In prreprocessing stage takes raw image as input and produce enhanced image as an output. In this study, there are different preprocessing algorithms used for image resizing, equalization, and noise removal. Histogram equalization, resising and noise filtering steps are performed so as to get the enhanced image.

\subsection{Eature extraction and performance evaluation}

Form enhanced image color features are extracted and from gray scale image texture and brisk local feature descriptor feature vectors are extracted. Once the representing features are extracted modeling of feed forward neural network had been done. In network modeling ReLU, sigmoid, tanh and gaussian transfer function had been used as a parameter for analyzing the performance. Finally, the performance of feed-forward neural network are tested. As shown Figure 2 the images are collected in day and night time to enhance and get different colors and finally they were resized in 256 by 256 pixel.

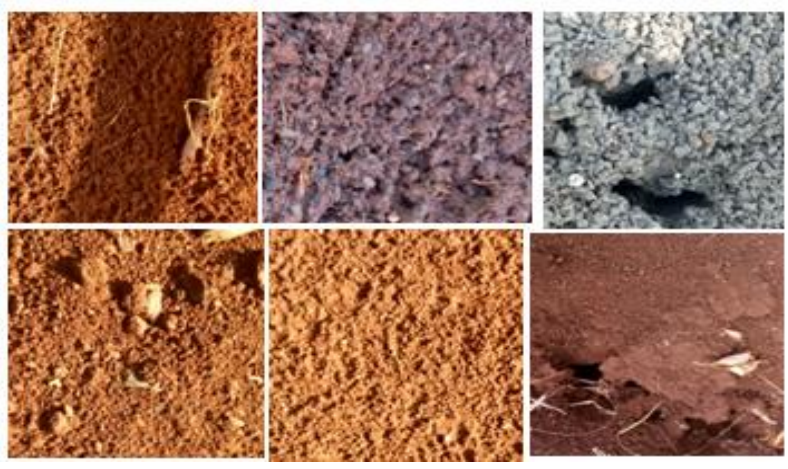

Figure 2. Sample images of soil classification

\section{RESULTS AND DISCUSSIONS}

An artificial neural network consists of three layers: the input layer (I), the hidden layer (H), and the output layer $(\mathrm{O})$. The input layer relies on as many neurons as input features. Input neurons just propagate input features to the hidden layer and the hidden layers process the input data. If there are errors while processing or learning patterns the errors are propagated to the hidden layers and computed again. Finally if the network is converged, the output is generated.

As indicated in Figure 3, the network needs color, texture, and brisk local feature as an input vector of the combined feature vectors and 4 hidden layes. In the hidden layers 26 neurons are used. The hidden layer has 26 neurons. This number was picked by trial and error methods, if the network has trouble of learning capabilities, and then neurons can be added to this layer. There is a significant change when we increase the number of hidden layers neurons until 21, 24 and 26 but there is no change when the number of hidden layer neurons increases above 26. In the output layers 9 neurons are considered to classify soils as Clay, Clay Sand, Silt Sand, Peat, Clay peat and moisture prediction as wet, dry and water. The next experiment is finding suitable transfer function for FFNN.

To achieve the goal of this study, the experimental scenario is conducted by considering the four transfer function of FFNN. In sigmoid transfer function, it maps the input to a value between 0 and 1 . Besides, the sigmoid's natural threshold is 0.5 , meaning that any input that maps to a value above 0.5 will be considered as 1 . The training time to converge the network as well as the accuracy while considering sigmoid function is lower than the other due to the range of transfer function. Since the range to map the input to is between -1 and 1 a better result has been achieve when tanh function is considered. Here, as shown in Figure 4, both the confergence rate and accuracy are better that sigmoid. In ReLU, there is a positive bias in the network for the layers in FFNN, as the mean activation is larger than zero. From the experiment we noticed that ReLU has better convergence and accuracy than sigmoid and tanh transfer function. In Gaussian transfer function the output is interpreted based on the degree of membership which contains a continuous degree of membership. The main reason that Gaussian transfer function has a better result is, it contains the continuous value for the 6 types of soil. The degree of membership of soil type 0 (clay soil) is between 0 and 1; for soil type 1(clay sand) the degree of membership is between 1 and 2 . When you see this the degree of membership is continuous and contains wider range. Finally from the experiment $97.4 \%$ accuracy is achieved. But, it has lower convergence rate than ReLU.

The effects of multiple layers feed-forward neural network transfer function in... (Belete Biazen Bezabeh) 


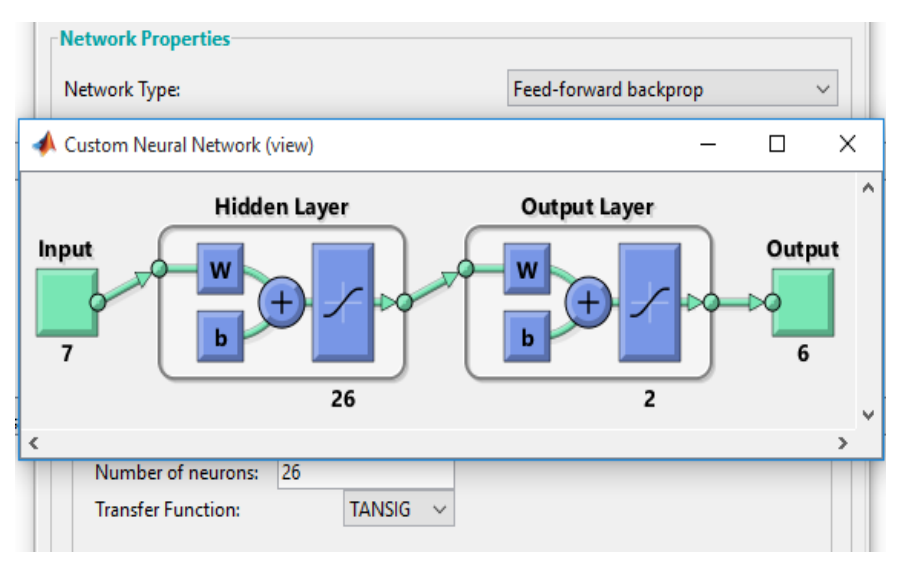

Figure 3. FFNN

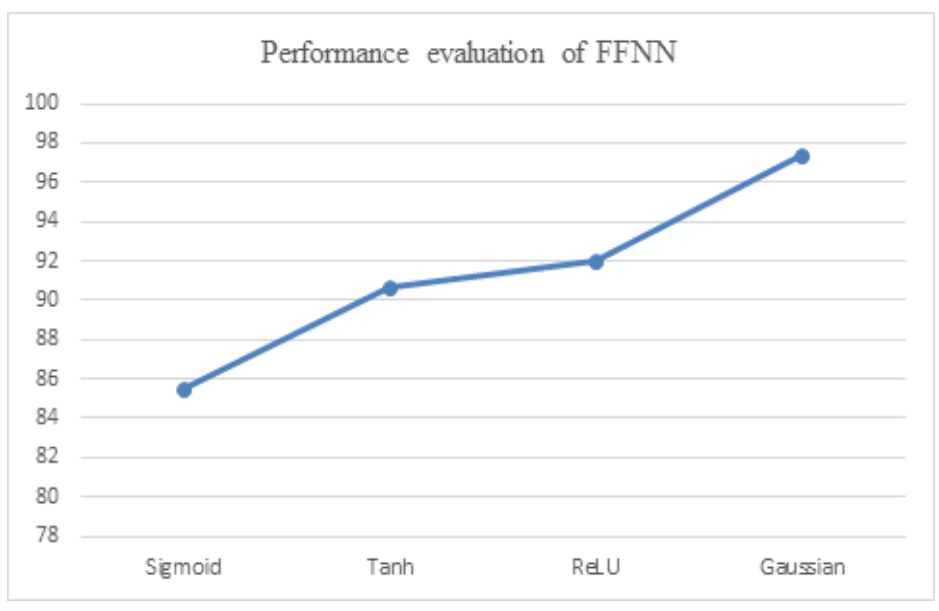

Figure 4. Result

\section{CONCLUSION}

The aim of this research paper is to identify suitable transfer function for classification and moisture level prediction using FFNN. In this paper, papametrs of FFNN are tested and the accuracy of the system is presented and the results of FFNN were discussed. The work can also be seen in depth and researched to analyze the other learninig function and using different machine learning techniques like deep learning in connection with soil in agriculture.

\section{ACKNOWLEDGEMENTS}

We gratefully acknowledge Bahir Dar University, Bahir Dar institute of Technology.

\section{REFERENCES}

[1] Mengistu Alemayehu, "Country Pasture/Forage Resource Profiles," Unpublished FAO, 2010.

[2] Kshitija S. Naphade, "Soil characterization using digital image processing," Lehigh University, Unpublished Theses and Dissertations, 1999.

[3] Tinku Acharya and Ajoy K. Ray, "Image Processing Principles and Applications," A John Wiley \& Sons, Publication, 2005.

[4] David C. Marvin, et al., "Integrating technologies for scalable ecology and conservation," Global Ecology and Conservation, vol. 7, pp. 262-275, 2016.

[5] Mingyuan Zhang, Tianzhuo Cao and Xuefeng Zhao, "Applying Sensor-Based Technology to Improve Construction Safety Management," MDPI Sensors, vol.17, no. 8, pp. 1841, 2017.

[6] Pravat Kumar Shit, Gouri Sankar Bhunia, and amkrishna Maiti, "Soil crack morphology analysis using image processing techniques," Modeling Earth Systems and Environment, vol. 1, no. 4, pp. 35, 2015. 
[7] K. Srunitha, and S. Padmavathi, "Performance of SVM classifier for image based soil classification," International Conference on Signal Processing, Communication, Power and Embedded System, IEEE, pp. 411-415, 2016.

[8] Dagnachew Melesew, and Abrham Debasu, "Soil Characterization and Classification: A Hybrid Approach of Computer Vision and Sensor Network," International Journal of Electrical and Computer Engineering (IJECE), vol. 8, no. 2, pp. 2088-8708, 2018.

[9] Ashok Kumar Patel and Snehamoy Chatterjee, "Computer vision-based limestone rock-type classification using probabilistic neural network,” Science Direct, vol. 7, no. 1, pp. 53-60, 2016.

[10] Anastasia Sofou and Georgios Evangelopoulos, "Soil Image Segmentation and Texture Analysis: A Computer Vision Approach," IEEE Geoscience and Remote Sensing Letters, vol. 2, no. 4, pp. 394-398, 2005.

[11] Sun-Ok Chung, et al., "Soil Texture Classification Algorithm Using RGB Characteristics of Soil Images," vol. 43, no. 26, pp. 34-38, 2010.

[12] Małgorzata Charytanowicz, Piotr Kulczycki, "An Image Analysis Algorithm for Soil Structure Identification," Springer, Intelligent Systems, pp. 681- 692, 2014.

[13] Bhawna J. Chilke, Neha B. Koawale and Divya M. Chandran, "Determination of Soil pH by using Digital Image Processing Technique-A Review," IJRITCC, pp. 501-503, 2017.

[14] Ali M. Ghalib, Roman D. Hryciw, and Seung Cheol Shin, "Image texture analysis and neural networks for characterization of uniform soils," Proceedings of the International Computing Congress on Computing in Civil Engineering, pp. 671-682, 1998.

[15] Umesh Kamble, et al., "Testing of Agriculture Soil by Digital Image Processing," IJSRD, 2017.

[16] A.V. Bilgili, W. et al., "Wavelet Analysis of Soil Reflectance for the Characterization of Soil Properties," 2008. [Online]. Available: http://conference.ifas.ufl.edu/SSC/pdf/BilgiliA.pdf.

[17] M. Barber, et al., "A novel method for 2-d agricultural soil roughness characterization based on a laser scanning technique," IEEE, vol. 2, pp. 731-733, 2008.

[18] Richard J. Flavel, et al., "An image processing and analysis tool for identifying and analysing complex plant root systems in 3D soil using non-destructive analysis Root1," PLoS One, 2017, Refered from, vol. 12, no. 5, 2017. [Online]. Available: https://doi.org/10.1371/journal.pone.0176433.

[19] Masayuki Tamura and Weiping Li, "Detection of soil liquefaction areas in the Kantou region using multi-temporal InSAR coherence," Synthetic Aperture Radar (APSAR), IEEE, pp. 548-551, 2013.

[20] Mrutyunjaya R. Dharwad, et al., "Estimation of Moisture Content in Soil Using Image Processing," International Journal of Innovative Research \& Development, 2014.

[21] Sanjay Kumawat, et al., "Sensor Based Automatic Irrigation System and Soil pH Detection using Image Processing," International Research Journal of Engineering and Technology, vol. 4, no. 4, pp. 3673-3675, 2017.

[22] Oliviu Matei, et al., "A Data Mining System for Real Time Soil Moisture Prediction," Procedia Engineering, vol. 181, pp. 837-844, 2017.

[23] Jase D. Sitton, Yasha Zeinali, and Brett A., "Story, Rapid soil classification using artificial neural networks for use in constructing compressed earth blocks," Construction and Building Materials, vol. 138, pp. 214-221, 2017.

[24] V. Rajeswari and K. Arunesh, "Analysing Soil Data using Data Mining Classifiation Techniques," Indian Journal of Science and Technology, vol. 9, no. 19, 2016.

[25] Sofianita Mutalib, S-N-Fadhlun Jamian, and Shuzlina Abdul-Rahman, "Soil classification: An application of self organising map and k-means. Intelligent Systems Design and Applications (ISDA)," International Conference, IEEE, pp. 439-444, 2010.

[26] Hamidreza Hassannejad Mohammad Sirus Pakbaz, and Rasool Mehdizadeh, "Comparison and Evaluation of Artificial Neural Network (ANN) Training Algorithms in Predicting Soil Type Classification," Bulletin of Environment, Pharmacology and Life Sciences, vol. 4, no. 1, pp. 212-218, 2015

[27] J.W. Hummel, K.A. Sudduth, and S.E. Hollinger, "Soil moisture and organic matter prediction of surface and subsurface soils using an NIR soil sensor" Computers and Electronics in Agriculture, Elsevier, vol. 32, no. 2, pp. 149-165, 2001.

[28] B. Bhattacharya, D.P. Solomatine, "Machine learning in soil classification," Neural Networks, vol. 19, no. 2, pp. 186-195, 2006.

[29] K. Kaviarasu, P. Sujith and G. Ayaappan, "Prediction of rainfall using image processing," IEEE, International Conference on Computational Intelligence and Computing Research, 2010.

[30] V. Atluri, Chih-Cheng Hung, and T.L. Coleman, "An artificial neural network for classifying and predicting soil moisture and temperature using Levenberg-Marquardt algorithm," IEEE Proceedings, pp. 10-13, 1999.

[31] K. K. Saju, "Classification of Macronutrient Deficiencies in Maize Plant Using Machine Learning," International Journal of Electrical and Computer Engineering (IJECE), vol. 8, no. 6, pp. 2088-8708, 2018

[32] Abrham Debasu, Dagnachew Melesew, "Soil characterization and classification using a hybrid approaches of computer vision and sensor network approach," International Journal of Electrical and Computer Engineering (IJECE), vol. 8, no. 2, pp. 989-995, 2018

[33] Hussein Attya Lafta, Zainab Falah Hasan, and Noor Kadhim Ayoob, "Classification of medical datasets using back propagation neural network powered by genetic-based features elector," International Journal of Electrical and Computer Engineering (IJECE), vol. 9, no. 2, pp. 1379-1384, 2019. 